\title{
In Situ TEM Straining of Ultrafine-grained Aluminum Films of Different Textures Using Automated Crystal Orientation Mapping.
}

\author{
Ehsan Izadi ${ }^{1}$, Amith Darbal ${ }^{2}$, Pedro Peralta ${ }^{1}$ and Jagannathan Rajagopalan ${ }^{1}$. \\ 1. Department of Mechanical and Aerospace Engineering School for Engineering of Matter, Transport \\ and Energy, Arizona State University, Tempe, Arizona, USA. \\ 2. AppFive LLC, Tempe, Arizona, USA.
}

Several studies have shown that metal films with similar thickness and grain size but dissimilar texture show significant differences in their mechanical behavior. For instance, ultrafine-grained (UFG) Al films with no preferred texture show lower flow stress and more pronounced nonlinear behavior during unloading compared to films with a bicrystalline microstructure. [1] [2] However, no systematic study has been focused on understanding the deformation mechanisms responsible for such differences in mechancial behavior.

In this study, two Al films, $200 \mathrm{~nm}$ thick and having a mean grain size of $\sim 250 \mathrm{~nm}$, were deposited on $200-\mu \mathrm{m}$ thick, $100 \mathrm{~mm}$ diameter, (100) silicon wafers using DC magnetron sputtering. For the first $\mathrm{Al}$ film, the Si wafer was etched with hydrofluoric acid to remove the native silicon dioxide layer before deposition, which resulted in a strong (110) out-of-plane texture with only two in-plane grain variants (bicrystalline film). For the second Al film, the silicon dioxide layer was left intact, leading to fully polycrystalline film with no preferred texture (non-textured film). Dog-bone shaped freestanding samples of the two films were then co-fabricated with micro-electro-mechanical systems (MEMS) based tensile testing devices using microfabrication techniques [3]. The MEMS devices are compatible with standard TEM straining specimen holders and have built-in force and displacement sensors that allow the measurement of the macroscopic stress and strain during in situ straining (Fig. 1).

In order to understand the texture-induced differences in mechanical behavior of non-textured and bicrystalline UFG Al films and to reveal the underlying deformation mechanisms, in situ TEM techniques are highly suitable. Previous in situ TEM deformation studies have usually focused on a combination of HRTEM and BF/DF TEM imaging to investigate different deformation mechanisms such as dislocation processes, twinning or grain boundary migration in a few well oriented grains. However, a statistically meaningful evaluation of these results is often difficult.

As an alternative approach, automated crystal orientation mapping in TEM (ACOM-TEM) has been used for crystallographic analysis. The main advantages of this approach include a much better identification of grains, more accurate detection of grain boundaries and direct orientation information over micron sized areas composed of several hundred grains. BF/DF TEM and HRTEM imaging, in contrast, can typically provide such information only for very few grains.

The ACOM results show that significant grain rotations, up to $\sim 6^{\circ}$, occur in the non-textured films even at small strains $(\sim 0.5 \%)$ during loading (Fig. 2), whereas the grains in the bicrystalline film exhibited significantly smaller rotations. Furthermore, reverse rotation of the grains occurred upon unloading in the non-textured film, which provides a possible reason for the observed nonlinearity in the stress-strain response (Fig. 2c). Bright field TEM imaging showed that grain contrast changes (indicative of grain rotations) were time dependent, which suggests that diffusive processes could be active in addition to 
dislocation slip. Therefore, the results show that the combination of ACOM and in situ TEM straining can provide a more detailed picture of the complex deformation processes occurring in UFG and nanocrystalline metals.

\section{References:}

[1] J. Rajagopalan and T. A. Saif, Journal of Materials Research, 26, (2011), p. 2826-2832.

[2] E. Izadi and J. Rajagopalan, Scripta Materialia, 114, (2016), p. 65-69.

[3] J. Han and M. Saif, Review of Scientific Instruments, 77, (2007), p. 045102.

[4] The authors acknowledge the use of facilities at the John M. Cowley Centre for High Resolution Electron Microscopy and the Centre for Solid State Electronics Research at Arizona State University.
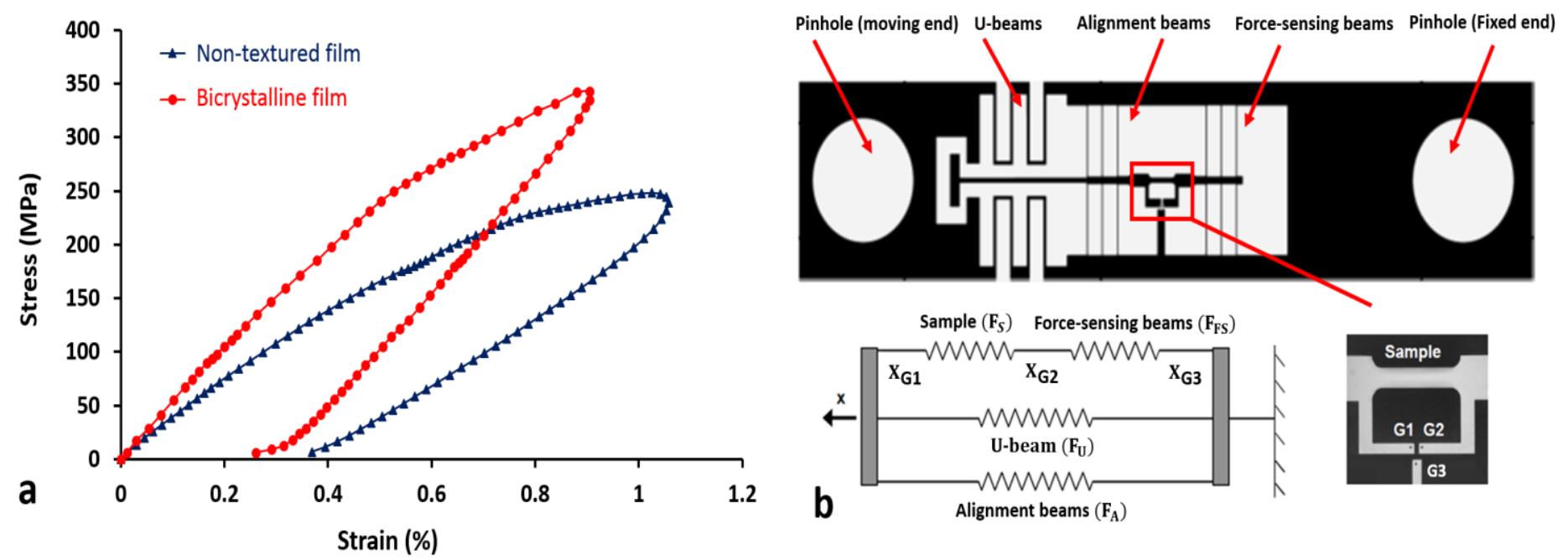

Figure 1. a) Stress-Strain response of bicrystalline and non-textured UFG Al film subjected to loadingunloading quasi-static tensile deformation. b) The MEMS based tensile testing device compatible with TEM straining specimen holders.
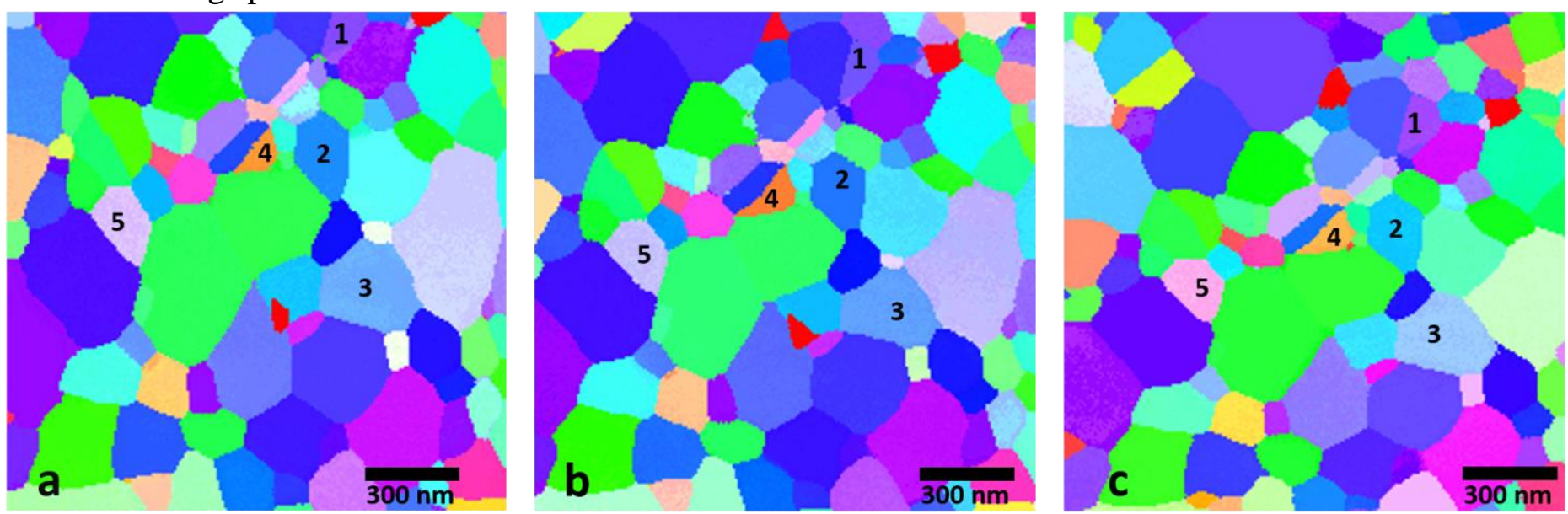

Figure 2. The results of orientation map for non-textured $\mathrm{Al}$ film at $0.5 \%$ strain during loading (a), $0.7 \%$ strain during loading (b) and $0.5 \%$ strain during unloading (c). The numbers show grains with notable rotations during loading and unloading. 